malignancy: A prospective cohort study protocol. Medicine (Baltimore). 2017;96(26):e7297.

[2] Schillaci G, et al. Aortic stiffness is increased in polymyalgia rheumatica and improves after steroid treatment. Ann Rheum Dis 2012;71:1151-6.

Disclosure of Interests: None declared

DOI: 10.1136/annrheumdis-2019-eular.4987

\section{THU0298 INITIAL MANIFESTATIONS AND OUTCOME OF PATIENTS WITH INCOMPLETE BEHÇET'S SYNDROME}

Sinem Nihal Esatoglu ${ }^{1}$, Seyda Bilgin ${ }^{2}$, Cem Sulu $^{2}$, Vedat Hamuryudan $^{3}$, Zekayi Kutlubay ${ }^{4}$, Serdal Ugurlu ${ }^{3}$, Emire Seyahi ${ }^{3}$, Melike Melikoglu $^{3}$, Izzet Fresko ${ }^{3}$ Sebahattin Yurdakul ${ }^{3}$, Hasan Yazici ${ }^{3}$, Gulen Hatemi ${ }^{3} .{ }^{1}$ Istanbul UniversityCerrahpasa, Cerrahpasa Medical Faculty, Department of Internal Medicine, Division of Rheumatology, Istanbul, Turkey, ${ }^{2}$ Istanbul University-Cerrahpasa, Cerrahpasa Medical Faculty, Department of Internal Medicine, Istanbul, Turkey, ${ }^{1}$ Istanbul University-Cerrahpasa, Cerrahpasa Medical Faculty, Department of Internal Medicine, Division of Rheumatology, Istanbul, Turkey; ${ }^{4}$ Istanbul UniversityCerrahpasa, Cerrahpasa Medical Faculty, Department of Dermatology, Istanbul, Turkey

Background: Behçet's syndrome (BS) has a heterogeneous expression involving many organ systems and is diagnosed by recognizing the coexisting manifestations. Several patients initially have few of these manifestations and do not fulfill the International Study Group (ISG) criteria When these are major organ manifestations, failure to recognize BS and treat promptly may lead to permanent damage in these organs.

Objectives: The aim of this study is to highlight the magnitude of this problem by surveying the frequency, presentation patterns and outcome of patients who did not fulfill ISG criteria when they presented to our clinic, but were followed and treated for manifestations strongly suggesting BS.

Methods: We conducted a retrospective chart review of all BS patients who were registered between 2003 and 2008. Among these 2385 patients, $199(8 \%)$ BS patients who did not fulfill ISG criteria at their initial visit were included in this study. Patients were called and a standard form was used for collecting demographic characteristics, BS manifestations at initial visit and during follow-up and treatment.

Results: Among the 199 patients (M/W: 90/109, mean age: $34 \pm 11$ years) who did not fulfill ISG criteria when they presented to our clinic, $70(35 \%)$ had major organ involvement. The types of major organ involvement that led to a diagnosis of BS at initial visit despite not fulfilling ISG criteria were eye involvement in 37, vascular involvement in 29 (venous thrombosis in 22, arterial aneurysms in 7), nervous system involvement in 3 and gastrointestinal (Gl) involvement in 1 patient. Thirtyfive patients $(18 \%)$ had a family history of BS

Of 199 patients, 167 had at least one more visit with a median follow-up of 11 years (IQR: 7-12). We were able to contact 116 of these patients and saw that 53 had fulfilled ISG criteria in the meantime. Among the 51 patients that we were not able to contact, 17 had fulfilled criteria while they were being followed in our clinic. Thus, a total of $70(42 \%)$ patients fulfilled ISG criteria after a median follow-up of 1.5 years (IQR: 1-4.25). All but 2 patients who developed eye involvement during the follow-up had fulfilled ISG criteria with a new mucocutaneous manifestation After a median follow-up of 4 years (IQR: 1-7), 23 (14\%) patients had developed at least one non-criteria BS manifestation, including vascular involvement in 10, arthritis in 13, neurologic involvement in 2 patients and $\mathrm{Gl}$ involvement in 1 patient.

Among the 81 patients who developed at least one new manifestation, $16(20 \%)$ were under immunosuppressive or interferon-alpha treatment at the time they developed their new manifestation. The remaining 65 patients had only received colchicine.

Among the 29 patients who had vascular involvement at initial visit, 7 (24\%) had a vascular relapse at different vascular site.

Three patients had another rheumatologic diagnosis (ankylosing spondylitis, seropositive rheumatoid arthritis and sarcoidosis) at the end of the follow-up.

Conclusion: In our 10-year follow-up cohort study, $42 \%$ of the 167 incomplete BS patients had fulfilled ISG criteria within a median duration of 1.5 years. Sixty-two (37\%) of these had major organ involvement that could have caused severe morbidity and mortality if the diagnosis of BS was missed and patients were untreated.

Disclosure of Interests: : Sinem Nihal Esatoglu: None declared, Seyda Bilgin: None declared, Cem Sulu: None declared, Vedat Hamuryudan
Consultant for: Abbvie, Amgen, BMS, Jansen, MSD, Pfizer, UCB, Speakers bureau: Abbvie, Amgen, BMS, Jansen, MSD, Pfizer, UCB, Zekayi Kutlubay: None declared, Serdal Ugurlu: None declared, Emire Seyahi: None declared, Melike Melikoglu: None declared, Izzet Fresko: None declared, Sebahattin Yurdakul: None declared, Hasan Yazici: None declared, Gulen Hatemi Consultant for: Abbvie, Amgen, BMS, Janssen, MSD, Pfizer, UCB, Speakers bureau: Abbvie, Amgen, BMS, Jansen, MSD, Pfizer, UCB,

DOI: 10.1136/annrheumdis-2019-eular.4287

\section{THU0299 PATIENT GLOBAL ASSESSMENT OF DISEASE ACTIVITY IN BEHÇET'S SYNDROME: A MULTICENTER STUDY}

Alberto Floris ${ }^{1}$, Matteo Piga ${ }^{1}$, Gerard Espinosa ${ }^{2}$, Nikolaos Kougkas $^{3}$, Andrea Lo Monaco $^{4}$, Giuseppe Lopalco ${ }^{5}$, Ida Orlando ${ }^{6}$, Vittorio Pirani ${ }^{7}$,

Ernestina Santos ${ }^{8,9}$, Luísa Serpa Pinto ${ }^{10}$, George Bertsias ${ }^{3}$, Luca Cantarini ${ }^{6}$, Alberto Cauli ${ }^{1}$, Ricard Cervera ${ }^{2,2}$, João Correia ${ }^{10}$, Marcello Govoni ${ }^{4}$, Florenzo lannone ${ }^{5}$, Ana Martins Da Silva ${ }^{8,9}$, Piergiorgio Neri ${ }^{11}$

Carlos Vasconcelos ${ }^{9}$, Alessandro Mathieu'. ' ${ }^{1}$ University of Cagliari, Rheumatology, Monserrato, Italy; ${ }^{2}$ University of Barcelona, Department of Autoimmune Diseases, Barcelona, Catalonia, Spain; ${ }^{3}$ University of Crete, Rheumatology, Clinical Immunology and Allergy, Heraklion, Greece; ${ }^{4}$ University of Ferrara, Rheumatology, Ferrara, Italy; ${ }^{5}$ University of Bari, Rheumatology, Bari, Italy; ${ }^{6}$ University of Siena, Rheumatology, Siena, Italy; ${ }^{7}$ Università Politecnica delle Marche, Ophthalmology, Ancona, Italy; ${ }^{8}$ Centro Hospitalar do Porto/Hospital de Santo António, Neurology, Porto, Portugal; ${ }^{9}$ University of Porto, UMIB Abel Salazar Biomedical Sciences Institute, Porto, Portugal; ${ }^{10}$ Hospital Santo Antonio Centro Hospitalar do Porto, Unidade de Imunologia Clinica, Unidade de Imunologia Clinica, Porto, Portugal;

${ }^{11}$ Cleveland Clinic Abu Dhabi, Eye Institute, Abu Dhabi, United Arab Emirates

Background: Disease activity evaluation is mandatory according to the OMERACT Core Set of Domains for Behçet's Syndrome (BS). Poor data are available on Patient (PtGA) and physician (PGA) global assessment of disease activity in BS.

Objectives: To assess PtGA performance in patients with BS and how different disease manifestations influence the patient and physician's perception of disease activity.

Methods: A multicenter cross-sectional cohort of consecutive BS patients was enrolled. Disease activity was evaluated by PtGA, PGA and the Behçet's disease current activity form (BDCAF). PtGA and PGA were assessed trough a single question ("How active was BS during the last week?") in a $10-\mathrm{cm}$ visual analogic scale. Health related quality of life (HRQoL) perception was evaluated by the Physical Component Summary (PCS) and the Mental Component summary (MCS) of the SF36v2 questionnaire.

Correlation between PtGA, PGA, BDCAF and between them and SF36v2 component summaries was assessed trough the Spearman's correlation coefficient (rho). Two separated multiple regression models were built to measure the independent effect ( $\beta$ regression) of each active clinical manifestation on PtGA and PGA

Results: Overall, 226 BS patients from 5 Mediterranean Countries were enrolled. Out of them, $111(49.1 \%)$ were males and the median (IQR) age and disease duration were 46.9 (35.6-55.2) and 11.7 (5.9-20.8) years, respectively.

The median (IQR) value of PtGA, PGA and BDCAF were 2.0 (0.3-5.0) $1.0(0.0-3.0)$ and $3.0(0.0-5.0)$, respectively. PtGA significantly correlated (Figure) with both PGA (rho 0.759. $p<0.001$ ) and BDCAF (rho 0.523. p $<0.001$ ), in support of its construct validity. PtGA scores were significantly higher than PGA with a mean (SD) difference of 0.8 (1.8), suggesting a different weighing of disease activity between patients and physicians. Indeed, PtGA and PGA were differently influenced by active clinical manifestations (Table). PtGA was mainly dependent on active mucocutaneous ( $\beta$ 0.243), gastrointestinal $\left(\beta\right.$ 0.216) and ocular $\left(\begin{array}{ll}\beta & 0.209\end{array}\right)$ involvement whereas a major effect of ocular manifestations was observed in PGA ( $\beta$ 0.378). PtGA was correlated with worse HRQoL perception measured by PCS (rho -0.476. $p>0.001$ ) and MCS (rho -451 $p>0.001$ ).

Conclusion: PtGA seems to be a valid outcome measure in BS and should be considered as a separate outcome measure or better be included in composite index for overall disease activity evaluation.

Abstract THU299 - Table 1

PtGA 


\begin{tabular}{lccccc}
$\begin{array}{l}\text { Active } \\
\text { involvement }\end{array}$ & $\boldsymbol{\beta}$ & $\mathbf{p}$ & $\begin{array}{c}\text { Active } \\
\text { involvement }\end{array}$ & $\boldsymbol{\beta}$ & $\mathbf{p}$ \\
\hline Mucocutaneous & 0.243 & $<0.001$ & Ocular & 0.378 & $<0.001$ \\
Gastrointestinal & 0.216 & 0.001 & Mucocutaneous & 0.284 & $<0.001$ \\
Ocular & 0.209 & 0.001 & Gastrointestinal & 0.192 & $<0.002$ \\
Vascular & 0.108 & 0.086 & Vascular & 0.129 & $<0.027$ \\
Arthritis & 0.088 & 0.185 & Arthritis & 0.121 & 0.048 \\
Neurologic & 0.003 & 0.962 & Neurologic & 0.047 & 0.420 \\
\hline
\end{tabular}

Disclosure of Interests: Alberto Floris: None declared, Matteo Piga: None declared, Gerard Espinosa: None declared, Nikolaos Kougkas: None declared, Andrea Lo Monaco: None declared, Giuseppe Lopalco Speakers bureau: SOBI, BMS, Ida Orlando: None declared, Vittorio Pirani: None declared, Ernestina Santos: None declared, Luísa Serpa Pinto: None declared, George Bertsias: None declared, Luca Cantarini: None declared, Alberto Cauli: None declared, Ricard Cervera: None declared, João Correia: None declared, Marcello Govoni: None declared, Florenzo lannone Consultant for: $\mathrm{F}$ lannone has received consultancy fees and/or speaker honoraria from Pfizer, AbbVie, MSD, BMS, Novartis, Lilly, UCB outside this work, Speakers bureau: $F$ lannone has received consultancy fees and/or speaker honoraria from Pfizer, AbbVie, MSD, BMS, Novartis, Lilly, UCB outside this work, Ana Martins da Silva: None declared, Piergiorgio Neri: None declared, Carlos Vasconcelos: None declared, Alessandro Mathieu: None declared

Figure. Correlation between PtGA and other outcomes measures
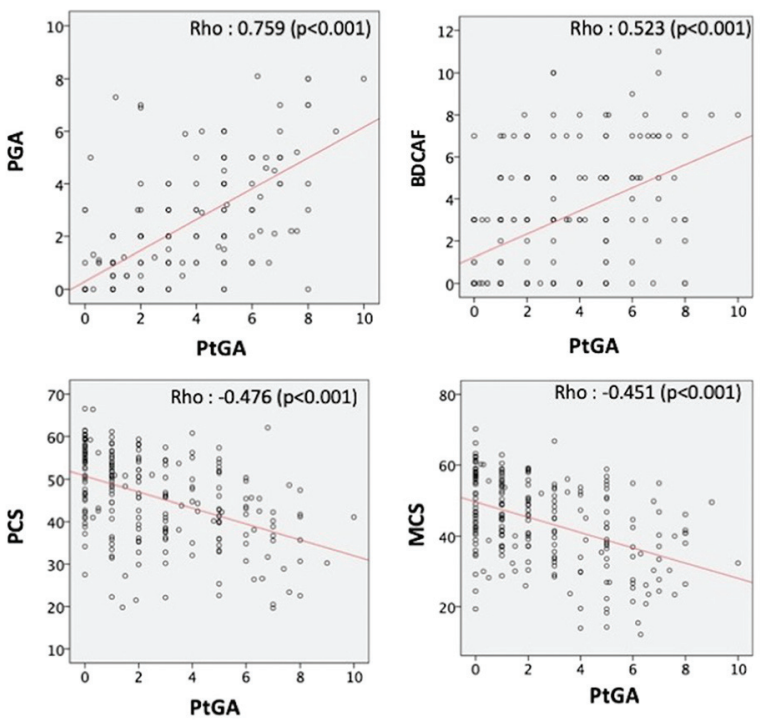

PtGA: patient's global assessment, PGA: physician's global assessment, BDCAF: Behçet's disease current activity form, PCS: physical component summary; MCS: mental component summary (MCS)

Abstract THU0299 - Figure 1

DOI: 10.1136/annrheumdis-2019-eular.2814

\section{THU0300 IMPACT OF VASCULAR ULTRASOUND ON EVALUATION OF GIANT CELL ARTERITIS AMONG 503 PATIENTS IN AN ACADEMIC MEDICAL CENTER}

Julia Ford ${ }^{1}$, Michael Diiorio ${ }^{1}$, William Docken ${ }^{1}$, Piotr Sobieszczyk ${ }^{2}$, Sara Tedeschi ${ }^{1}$. ${ }^{1}$ Brigham and Women's Hospital, Department of Medicine, Division of Rheumatology, Allergy and Immunology, Boston, United States of America; ${ }^{2}$ Brigham and Women's Hospital, Department of Medicine, Divison of Cardiovascular Medicine, Boston, United States of America

Background: As vascular ultrasound (VUS) is increasingly used in evaluation of giant cell arteritis (GCA), its impact on clinical care is of great interest.
Objectives: To describe utilization of VUS in GCA evaluation at a large academic medical center and investigate impact of VUS on clinica impression.

Methods: We performed a retrospective cohort study of patients who underwent VUS at a single center in Boston, 2013-2017, to evaluate suspected or known GCA. Trained cardiovascular ultrasound technicians used a standardized protocol to image the temporal, carotid, subclavian, and axillary arteries for presence of hypoechoic circumferential wall thickening (halo sign), hyperechoic wall thickening, stenosis, and occlusion. VUS images were interpreted by trained cardiovascular medicine physicians as consistent with acute arteritis ("acute"), no evidence of arteritis ("none"), or hyperechoic wall thickening without halo sign ("hyperechoic"). Demographic, laboratory, medication, pathology and clinical data including the treating physician's clinical suspicion for GCA pre- and post-VUS were obtained by electronic medical record review. Fisher's exact test and Wilcoxon rank-sum tests compared baseline characteristics among patients with VUS positive for acute arteritis ("acute") vs. negative for acute arteritis ("none" or "hyperechoic"). We compared the treating physician's preand post-VUS clinical suspicion for GCA among patients with no history of GCA or aortitis.

Results: We identified 503 patients with median age 70.4 years; $69.0 \%$ were female and $87.5 \%$ White. VUS interpretation was acute in 48 patients (9.5\%), none in 427 (84.9\%), and hyperechoic in 28 (5.6\%). Baseline characteristics are presented in Table 1. Weight loss, cranial symptoms, higher ESR, and steroid use were more common in patients with VUS positive for acute arteritis. Change in the treating physician's clinical suspicion for GCA is shown in Figure 1. VUS positive for acute arteritis increased suspicion for GCA in 26/35 (74\%) cases, and VUS negative for acute arteritis lowered suspicion for GCA in 303/396 (77\%) Of 110 patients with a temporal artery biopsy after VUS, $16(14.6 \%)$ biopsies showed active arteritis; 11 of these were in patients with VUS negative for acute arteritis.

Conclusion: VUS result changed the clinical suspicion for GCA in approximately $75 \%$ of patients in this large cohort.

Abstract THU300 - Table 1. Characteristics at the time of VUS

\begin{tabular}{|c|c|c|c|c|}
\hline Age, years & $\begin{array}{l}70.4(62.7 \\
77.5)\end{array}$ & $\begin{array}{l}73.2(65.8 \\
79.7)\end{array}$ & $\begin{array}{r}69.9 \\
(62.6, \\
77.3)\end{array}$ & NS \\
\hline History of GCA or aortitis & 14.3 & 27.1 & 13.0 & 0.02 \\
\hline History of PMR & 31.2 & 22.9 & 32.1 & NS \\
\hline New headache & 51.3 & 60.4 & 50.3 & NS \\
\hline Weight loss & 13.3 & 22.9 & 12.3 & $0.05^{* *}$ \\
\hline Fatigue & 36.2 & 45.8 & 35.2 & NS \\
\hline Jaw claudication & 10.9 & 33.3 & 8.6 & $<0.01$ \\
\hline Scalp tenderness & 17.1 & 37.5 & 15.0 & $<0.01$ \\
\hline $\begin{array}{l}\text { TA tenderness/decreased TA } \\
\text { pulse }\end{array}$ & 14.5 & 29.2 & 13.0 & $<0.01$ \\
\hline Transient vision loss & 7.8 & 10.4 & 7.5 & NS \\
\hline Permanent vision loss & 3.0 & 6.3 & 2.6 & NS \\
\hline $\mathrm{CRP}^{\star}, \mathrm{mg} / \mathrm{L}$ & $15.7(3.1,76.0)$ & $36.3(8.1,81.9)$ & $\begin{array}{l}13.7 \\
(2.9 \\
75.3)\end{array}$ & NS \\
\hline $\mathrm{ESR}^{\star}, \mathrm{mm} / \mathrm{h}$ & $40(18,83)$ & $67(30,95)$ & $\begin{array}{c}40 \\
(17 \\
81)\end{array}$ & $<0.01$ \\
\hline Steroids at time of VUS & 57.5 & 75.0 & 55.6 & 0.010 .02 \\
\hline$>0$ to $<15 \mathrm{mg} /$ day & 34.3 & 30.6 & 34.8 & NS \\
\hline$\geq 15$ to $<40 \mathrm{mg} /$ day & 23.5 & 8.3 & 25.7 & \\
\hline$>40 \mathrm{mg} / \mathrm{day}$ & 42.2 & 61.1 & 39.5 & \\
\hline Steroids $>3$ weeks & 56.8 & 41.7 & 59.3 & \\
\hline
\end{tabular}

Presented as\% or median (interquartile range)

CRP: C-reactive protein; ESR: erythrocyte sedimentation rate; GCA: giant cell arteritis; PMR: polymyalgia rheumatica; TA: temporal artery; VUS: vascular ultrasound. NS: not significant.

${ }^{*}$ Among 418 patients with CRP and 370 with ESR checked within 3 weeks before VUS

${ }^{* *} p=0.046$ 